\title{
La información, ¿abstracción o realidad? : Las paradojas de un concepto fundamental
}

\author{
Beatriz Gil Maza y Pedro C. Marijuán \\ Ingeniería Eléctrica, Electrónica y Comunicaciones \\ CPS, Universidad de Zaragoza (E 50015, Zaragoza)
}

\subsection{Resumen}

La información es un concepto polémico en las más diversas disciplinas. En el campo de la física, la idea de que información y entropía están íntimamente relacionadas ha circulado durante bastantes años. Sin embargo, los conceptos avanzados por Boltzmann y Schrödinger difieren notablemente de las hipótesis que Stonier plantea en su reciente libro "La información y la estructura interna del universo", donde reflexiona sobre la posibilidad de que la información sea una propiedad del universo, una parte de su estructura interna. En la teoría de la comunicación de Shannon, la información se asocia con el grado de libertad de elección que tenemos al construir los mensajes. Stonier defiende que la definición del término información es análoga a la definición que hacen los físicos del término energía. La energía se define como la capacidad para realizar un trabajo; a la información le correspondería la capacidad para organizar los sistemas. Al igual que existen diferentes formas de energía (mecánica, electromagnética, térmica, química...), podemos encontrar información de naturaleza muy diversa (información humana, información biológica, información genética...) y no debemos confundir la realidad de la información en sí misma, con la interpretación de la información en un determinado contexto. Se pueden proporcionar numerosos ejemplos de la necesidad, en el actual sistema científico, de un análisis riguroso de la información. En biología p. ej., existe la necesidad de avanzar en el conocimiento del sistema de señalización celular, la red metabólica, el sistema nervioso, etc., sistemas que proporcionan las dinámicas más sorprendentes, si atendemos a los diferentes mecanismos de procesamiento de la información que emplean; y que sólo parecen ofrecer posibilidades de ser estudiados (mediante una teoría integrativa) en el contexto de una teoría general de la información, o en una nueva ciencia emergente centrada en la información. (Autor)

Palabras clave: Información. Orden. Desorden. Energía. Entropía. Vacío funcional.

Scire. 2 : 1 (en.-jun. 1996). 


\subsection{Abstract}

The concept of information incites discussion in quite a few scientific realms. In physics, the relationship between information and entropy has been around for years. Information has been associated traditionally by communication engineers, with the freedom of choosing symbols to construct a message. To confuss matters further, Shannon used entropy as a metaphor in his analysis of statistical behavior of symbols. In contrast to communication engineers, Stonier, in his recent book "Information and the internal structure of the universe", points out the possibility of a different relationship between information and entropy. The author asserts that the definition of information should be analogous to the physical definition of energy. Energy is defined as the capability of performing work, whereas the capability of organizing systems corresponds to information. We can find different kinds of energy (electromagnetic, mechanic, thermal, chemical...), there exist as well different kinds of information (human, social, biological...). Evenmore, it is necessary to discern between the reality of information on its own, and the meaning of information inside each context. One of the most striking contexts are biological systems, which rely on sophysticated information procesing mechanisms not well-understood yet. (Author)

Key words: Information. Order. Disorder. Energy. Entropy. Functional void.

\section{Introducción: insuficiencia de las respuestas clásicas}

Vivimos en lo que se ha dado en llamar la era de la información. Anteriores términos como "era atómica" y "era espacial" han quedado claramente arrinconados en favor de este término que al parecer todos entendemos, pero que, como ocurre a veces con los conceptos mas fundamentales, nadie sabe explicar cuando se ha de dar respuesta cabal. No cabe duda que los computadores, la biología molecular y las telecomunicaciones, principales factores de cambio del mundo actual, tienen todos como "sustancia" propia (sea lo que sea) a la información.

Aunque teóricos de la termodinámica y la física ya se habían acercado al fenómeno informacional décadas antes: Boltzmann, Maxwell, o el mismo Schrödinger, el concepto de información no emerge con autoridad dentro del sistema de las ciencias hasta la postguerra: teoría de la información, cibernética, teoría de sistemas, teoría de juegos, los primeros computadores, la elucidación de la estructrura del ADN..., con la consiguiente avalancha de aplicaciones del concepto de información en muchos otros campos: biología molecular, neurociencias, lógica, lingüística, ecología, economía, etc. Sin embargo, al cabo de unas dos décadas, parece agotarse la dimensión interdisciplinar del término y paradójicamente es cuando se asiste a su entronización como exponente de la tecnología y la evolución social de nuestra época (Marijuán, 1996; este volumen).

Scire. 2 : 1 (en.-jun. 1996). 
Cronológicamente, cuatro grandes grupos de teorizaciones han intentado dar cuenta, desde aquellos años pioneros hasta la fecha, del fenómeno de la información:

- información termodinámica, clásicos como Boltzmann (1896), Schrödinger (1944), y otros autores contemporáneos como Prigogine (1985), Wicken (1987), Swenson, y Stonier (1990).

- transmisión de la información, Nyquist, Hartley, Shannon y Weaver (1964).

- información lógico-semantica, Popper (1993), Carnap, Bar-Hillel.

- informacion algorítmica, Kolmogorov, Chaitin.

Y aún se podría añadir una información autoorganizativa con autores como Kauffman (1993), Haken, los teóricos del caos y la no-linealidad, (Instituto de Santa Fe), etc. Sin embargo, a pesar de tan numerosos esfuerzos para el desarrollo de una teoría de la información no son pocos los autores que reconocen los escasos logros obtenidos, quizá resultantes de una ausencia general de coherencia entre los distintos planteamientos, lo que desde luego dificulta el desarrollo de una teoría integrativa (Anderson, 1972; Rosen, 1993).

Una de las visiones generales más sugerentes es la de Stonier (1990), quien en su libro recientemente vertido al español (Stonier, 1996), propone una teoría informacional que intenta contemplar desde los sistemas biológicos y sociales a la cosmología, proporcionando una excelente base para reanudar la reflexión sobre las posibilidades que ofrece una perspectiva informacional en el sistema de las ciencias. La discusión de su obra será uno de los elementos centrales del presente artículo.

\section{La realidad de la información}

"La información y la estructura interna del universo," título de la obra de Stonier, explora la creación de una nueva teoría general de la información — particularmente en el contexto de la física- centrando sus argumentos en la naturaleza de la información y su realidad en todo lo que nos rodea. Se trata de conjeturar sobre la realidad intrínseca de un concepto que de diversos modos nos presenta su efecto causal sobre cosas que han sido previamente aceptadas como reales.

El argumento fundamental se centra en que la percepción del mundo es un producto de nuestra experiencia histórica, y ésta no ha incorporado la información salvo con carácter de abstracción y perteneciente al "mundo interior", a nuestra propia mente, y no como una propiedad del Universo semejante a la materia o la energía. La paradoja inherente a los conceptos de masa y materia no

Scire. 2 : 1 (en.-jun. 1996). 
fue resuelta hasta que nuestra experiencia proporcionó los dispositivos adecuados para resolverla: los átomos no fueron aceptados como reales hasta que dejaron de ser átomos (Popper y Eccles, 1977). Nuestra situación actual en relación con la información es similar: la experiencia con las máquinas de la información data desde hace solamente cuarenta años; es razonable que después de la primera sorpresa ante estos dispositivos capaces de procesar la información, antes privilegio exclusivo del cerebro humano, nos planteemos ahora la existencia de la información, una "realidad" como la materia y la energía, y podamos desligarla definitivamente del sistema que la transmite, recibe, procesa o almacena (Stonier, 1990).

La aceptación de esta realidad no debe ser un gran problema, puesto que el objetivo final de todas las ciencias es la obtención de información del sistema al que se aplican. Además, en múltiples contextos de la física y otras ciencias se trabaja frecuentemente con distintas funciones basándose en el hecho de que contienen toda la información dinámica del sistema. No obstante, para reconocer como información cualquier cambio que suponga una disipación de energía necesitamos un observador que levante acta, (Margalef, 1995). El problema del observador se plantea con toda crudeza al intentar averiguar la naturaleza de la información, y sobre todo su "sentido". Obviando dicho problema en una primera aproximación, el argumento principal de las hipótesis de Tom Stonier, puede ser reducido a lo siguiente:

La información está intrínsecamente relacionada con la organización de un determinado sistema, y se puede describir como la causa eficiente del estado de organización de éste.

La información, como la energía, es una magnitud abstracta. En su interacción con la materia o la energía es cuando muestra su naturaleza, reorganizando o bien manteniendo el orden existente. Inherente a cada postulado y a cada ecuación de la física aparece el concepto de información. Las leyes de la física conducen total o parcialmente al conocimiento de la organización interna del sistema al que se aplican, y la organización es un reflejo del contenido en información del sistema. Avogadro, Boltzman, Planck describen esa organización como manifestación del orden que subyace en las constantes físicas. Desde Galileo describimos el movimiento en términos de distancia y tiempo; dado que el cambio de posición de una partícula supone un cambio en la organización del universo, consecuentemente se produce un cambio también en el contenido informacional del sistema que la contiene. Otros fenómenos como las transiciones de fase muestran con mayor evidencia el cambio en el orden macroscópico que se ha producido en el sistema: las roturas de simetría proporcionan información adicional al sistema, con el consiguiente aumento de complejidad.

Scire. 2 : 1 (en.-jun. 1996). 
La información forma parte de nuestro universo, es un componente implícito de cada ecuación que gobierna las leyes de la física, y manifiesta su efecto en su interacción con la matería y la energía. Si un sistema exhibe organización, contiene información. La organización no es sino el orden que presentan las partes constituyentes de un sistema. Orden es opuesto a desorden. En el contexto de la termodinámica, relacionado con el desorden, se habla de probabilidad, concepto que a través de una función muy especial, la entropía, nos permitirá relacionar orden con información.

\section{Información y entropía}

L. Boltzmann propuso en 1872 una interpretación microscópica de la entropía en términos de movimiento molecular, como una medida del "desorden" o número de microestados compatibles con un determinado macroestado. La entropía no sólo expresa el grado de mezcla de un sistema, sino que mediante la palabra asociada desorden se indican también aspectos tales como la indistinguibilidad de los elementos del sistema que estamos considerando, y la incertidumbre o simetrías que presenta el sistema, de modo que podemos hablar de ausencia de información acerca del microestado concreto en que efectivamente se encuentra el sistema. La relación existente entre desorden y falta de información constituye otro de los cimientos de las hipótesis de T. Stonier:

La entropía de un sistema puede ser alterada modificando el contenido en calor, o bien la organización del sistema. Ambos procedimientos ocasionan un cambio en el contenido informacional del sistema. La información se puede aproximar a una función exponencial inversa de la entropía:

$$
\mathrm{S}=\mathrm{k}^{*} \ln (\mathrm{D}) ; \mathrm{Or}=1 / \mathrm{D} ; \mathrm{I}=\mathrm{C}^{*} \mathrm{Or} ; \mathrm{I}=\mathrm{C}^{*} \exp (-\mathrm{S} / \mathrm{k}) ; \mathrm{I}=\mathrm{Io} * \exp (-\mathrm{S} / \mathrm{k})
$$

donde Io será la información a entropía cero $(\mathrm{S}=0)$ y de este modo (desorden) $\mathrm{D}=\mathrm{Io} / \mathrm{I}$, representando este cociente una funcion de probabilidad consistente con la de Boltzmann.

El punto principal de discusión radica en asumir el orden como inverso al desorden, de modo que podamos pasar de desorden, no-organización, entropía, a orden, organización, información. Independientemente de que esta relación sea inversa u opuesta, y de que el orden sea aditivo o multiplicativo, vemos que información y entropía deben presentar una relación antagónica, y que además la información es un concepto formal que podría ser aplicado en aquellos casos en que la formulación en términos de entropía no sea sencilla, como es la evolución de los sistemas abiertos o los sistemas biológicos.

La función de probabilidad de Boltzmann constituye una medida del desorden generado por el calor del sistema; no podemos aplicar la ecuación para examinar posibles estados de entropía menores que $S=0$, ni podemos tener menor

Scire. 2 : 1 (en.-jun. 1996). 
energía que el estado a $\mathrm{T}=0^{\circ} \mathrm{K}$.

El Postulado de Nerst o Tercer Principio de la Termodinámica debería ser modificado por la "fisica de la información", puesto que no hay nada que impida aumentar el grado de organización de un sistema añadiéndole información. El concepto de entropía negativa, poco plausible para el físico o el ingeniero, parece ser ampliamente aceptado entre los que intentan avanzar en el desarrollo de una teoría general de la información.

También Schrödinger propuso modificaciones a la ecuación de Boltzmann, enfatizando que el orden y el desorden no tienen limitaciones en entropía, de modo que siempre podemos conseguir mayor organización de un sistema, y por consiguiente una mayor disminución de entropía. Esto no constituye una provocación a las teorías cosmológicas, es sólo en sistemas cerrados donde la entropía no puede decrecer. El resto del universo puede estar evolucionando hacia un estado de máxima entropía, pero la entropía de este planeta (con la ayuda del sol) está decreciendo continuamente; los seres vivos está claro que evolucionan también en una dirección de creciente improbabilidad termodinámica.

Mientras que la física en los últimos años se ha centrado en el estudio de la evolución del Universo, en la Biología sucedía estrictamente lo opuesto. El esfuerzo se ha centrado aquí en la evolución de los sistemas complejos: sistemas simples que se configuran en sistemas más complejos, más integrados, más diferenciados; sistemas que se autoorganizan, constituyéndose cada vez más y más improbables termodinámicamente. La complejidad se sirve de la complejidad ya existente para conseguir un mayor grado de complejidad. Evidentemente no se puede pasar del Big-Bang a la máquina de vapor: para los organismos complejos multicelulares la célula procariota es un requisito previo, y tomó cerca de un billón de años a la evolución el pasar de los organismos unicelulares a los seres humanos. F. Jacob en El juego de lo Posible (1981), destaca la importancia de la adquisición de una nueva información en las pocas fases verdaderamente creadoras de la evolución: y cómo la especialización y la diversificación, aunque aparentemente sólo requieran una utilización distinta de la misma información estructural, proporcionan los caminos adecuados para seguir avanzando en esta trayectoria de complejidad que diríase no tiene límite.

\section{La célula viva como "motor" informacional}

La entropía, calculable sobre la irreversibilidad de las reaciones que tienen lugar en los organismos vivos o en el entorno que los rodea, puede ser recuperada aumentando la organización o bien la información del sistema: ésta puede ser una información dinámica, o bien potencial, como la información genética (Margalef, 1995).

Scire. 2 : 1 (en.-jun. 1996). 
Así, el estudio de la información en los sistemas biológicos nos remite de nuevo a las tesis de Stonier: fundamentalmente a la distinción entre información dinámica e información estructural. Este dualismo es semejante al existente entre los aspectos inercial y gravitacional de un sistema material, donde la relación entre masa inercial y masa gravitacional condujo a Einstein a su Principio de Equivalencia entre inercia y gravitación.

En lenguaje causal, la información dinámica adoptará el carácter de causa eficiente o fuerza que actúa sobre el sistema, frente a la información estructural, que adquiere el carácter de causa formal -los posibles estados del sistema constituirán las causas materiales de éste. Como ejemplo sencillo de este dualismo, surge el concepto biológico de fenotipo-genotipo. El genotipo, en el sentido de determinador de especie o de identidad, está asociado al fenotipo, el cual expresa dinámicamente la información estructural que le aporta el primero (Rosen, 1993).

Las relaciones entre el mundo de los organismos "reales" y los organismos "potenciales" no han podido precisarse verdaderamente, excepto en algunos rasgos sencillos, donde la correlación entre un gen determinado y su manifestación obedece a mecanismos simples. En la mayoría de los casos la situación es mucho más compleja, puesto que un mismo gen interviene en la expresión de numerosos caracteres y un mismo carácter puede estar controlado por numerosos genes. Estas estructuras que conectan las distintas realizaciones que se observan en los individuos, no sólo son muy numerosas y complejas, sino que funcionan a distintos niveles de integración.

$\mathrm{Si}$ atendemos ahora al funcionamiento del ciclo celular es necesario distinguir tres grandes subsistemas con un alto contenido en información estructural y cinética, estos hacen posible el desarrollo del ciclo vital del individuo multicelular: la red metabólica, el "mundo del ADN", y el sistema de señalización (también se podría distinguir como subsistema propio la maquinaria proteolítica), todos ellos basados en enzimas y proteínas específicas (Marijuán, 1996).

El funcionamiento de cada enzima es una auténtica maravilla en cuanto a eficiencia, especificidad, miniaturización y relaciones "respetuosas" con el medio. Las redes de enzimas asociadas con cada uno de los subsistemas anteriores sirven de soporte para la emergencia de una propiedad organizativa abstracta característica. Respectivamente las denominaremos:

- autoorganización

- automodificación

- autorreconfiguración

Estas propiedades están armoniosamente acopladas en relación con el desarrollo del ciclo vital de la célula, bien se trate de organismos unicelulares o mul-

Scire. 2 : 1 (en.-jun. 1996). 
ticelulares. El ciclo celular adquiere el carácter de "motor" del organismo y del proceso evolutivo. Se trata de un auténtico motor de solución de problemas organizativos, donde el papel del sistema de señalización quizás es el más llamativo desde el punto de vista del procesamiento de la información (en parte, porque es el que la biología molecular está explorando actualmente).

La información dinámica queda igualmente referenciada respecto al desarrollo del ciclo celular. Es éste quien dicta y orienta el procesamiento de la información incidente y su "contravalor entrópico", el vacío funcional, o conjunto de cambios introducidos en la célula a través del sistema de señalización. Dicho vacío funcional representa un estado de elevada información dinámica o cinética que desaparecerá en el instante que se restaure el equilibrio del sistema, transformándose esta información cinética en agitación térmica o bien en una reorganización del sistema, (información estructural).

Podríamos decir que los vacíos funcionales contienen la información a procesar por la célula; y dependiendo del tipo de procesamiento que ésta realice, llegaremos a un estadio organizativo o a otro diferente. Este tipo de procesamiento cuasi-universal hace posible la emergencia de la multicelularidad, organismos que son capaces de desarrollar procesos organizativos altamente sofisticados entre los que destaca, desde esta perspectiva informacional, el sistema nervioso.

\section{La evolución de los sistemas nerviosos}

Una neurona es una célula más y sus propiedades "informacionales" tan especiales no dejan de estar construídas a partir de los subsistemas generales celulares a que aludíamos antes. Lo que sí se aprecia en las neuronas es una enorme hipertrofia de su sistema de señalización y un alto grado de especificidad: son células especializadas en procesar la materia orgánica más sutil, la información.

Los sistemas nerviosos proporcionan una herramienta evolutiva de primer orden a los animales multicelulares. Se podría decir que empieza un nuevo proceso evolutivo gracias a ellos, sobre todo a partir de la crucial divergencia evolutiva entre protóstomos (invertebrados) y deuteróstomos (vertebrados). Se aprecia claramente en relación con el comportamiento y los propios sistemas nerviosos que es la coevolución la que plantea los problemas desde los ecosistemas, la autoevolución la que da respuestas desde la propia capacidad evolutiva del organismo, y la selección (darwinista) la que juzga o califica la adecuación entre las dos primeras (a veces, de forma bastante aleatoria). Un "superdarwinismo" como el que ha estado tan en boga en décadas pasadas (p.ej., el caso de la sociobiología) pierde de vista las dos terceras partes del problema, si no más.

La propia evolución del sistema nervioso de los vertebrados es un curiosa escenificación de la tesis anterior. La feroz competencia en el ecosistema diurno

Scire. $2: 1$ (en.-jun. 1996). 
del Triásico entre los antecesores mamíferos y los reptiles no fue favorable para los primeros; sin embargo, una serie de respuestas autoevolutivas sumamente adecuadas respecto al nicho nocturno dieron como resultado una línea de procesamiento en el córtex cerebral superior al de otras especies. Los mamíferos, gracias a sus nuevas capacidades cerebrales, lograron imponerse en la mayor parte de los ecosistemas (Jerison, 1976). Algunas de las cualidades que apreciamos como únicas en el género humano, ya quedan esbozadas a partir de aquellas lejanas contiendas evolutivas del Jurásico. Es curioso que el control del ciclo de la neurogénesis haya sido la herramienta más importante en estos casos de "autoalteración" evolutiva del sistema nervioso.

En general, el sistema nervioso ha de ser entendido como un sistema de señalización, de procesamiento y registro de la información a la escala del conjunto del organismo. Por medio de su computación basada en "mapas" y moléculas químicas (hormonas, neuropéptidos, neurotransmisores) adapta el comportamiento a las necesidades del ciclo vital. De nuevo encontramos un encadenamiento estrecho entre el procesamiento de la información y el ciclo vital del individuo .

Quizá la teoría integradora acerca del funcionamiento del sistema nervioso que más matiza lo referente al procesamiento de la información respecto a las necesidades de un ciclo vital, es la teoría dual de K.P. Collins, que integra los hallazgos de diversas ramas de las neurociencias experimentales, y considera las columnas celulares como las unidades genuinas en cuanto a morfología, desarrollo y procesamiento de la información en el córtex vertebrado (Marijuán, 1996).

\section{Conclusión}

La información se ha disuelto en los anteriores sistemas biológicos, enzima, célula, sistema nervioso, en una red de procesos que genuinamente "intentan" llenar los vacíos o necesidades existentes respecto a los ciclos vitales o de funcionamiento de los organismos. Si contemplamos los propios aspectos informacionales de nuestras sociedades contemporáneas, también se puede apreciar una congruencia para con lo anterior. Como ya planteaba el teórico de los medios de comunicación Marshal McLuhan, la información social es enigmática, tiende a estar centrada más en las ausencias que en las situaciones normales en sí: "real news are bad news" (Marijuán, 1996b).

Quizá no sea acertado pretender una reducción fisicalista de la información e intentar obtenerla a partir de otros conceptos físico-matemáticos. Una teoría general de la información parece que debe ser contemplada como una nueva perspectiva en sí misma, base de una nueva ciencia "vertical" en ciernes. Tom Stonier $(1990,1996)$ sostiene que el papel social y científico de los ordenadores

Scire. $2: 1$ (en.-jun. 1996). 
guarda un estrecho paralelismo con lo que ocurrió hace dos siglos con las máquinas de vapor. Estas no solo propiciaron una revolución industrial, económica y social, sino cambios científicos fundamentales. Unas generaciones más tarde de la definitiva extensión social de las máquinas de vapor, éstas dieron lugar al surgimiento de una nueva ciencia: la Termodinámica. Primero se produjo la experimentación técnica, luego vino la reflexión científica...

\section{Referencias bibliográficas}

Anderson, P.V. (1972). More is Different: Broken Symmetry and the Nature of the Hierarchical Structure of the Science. // Science. 177 (1972) 393-396.

Boltzmann, L. (1896). Lectures on Gas Theory. Berkeley, California : University of California Press, 1976.

Brillouin, L. (1956). Science Information Theory. New York Academic Press, 1956.

Jacob, F. (1982). El Juego de lo Posible. Barcelona : Grijalbo, 1982.

Jerison, H. (1976). Paleoneurology and the evolution of mind. // Scientific American. 234 (1976) 90-101.

Kauffman, S. A. (1993). The Origins of Order. New York : Oxford University Press, 1993.

Mainzer, K. (1988). Symmetries in Nature. // CHIMIA, 42 : 5 (1988) 161-171.

Matsuno, K.(1978). A Theoretical Basis of Margalef's Principle in Ecosystems. // Journal of Theoretical Biology. 70 (1978) 23-31.

Marijuán, P. C. (1996). Information and Symmetry in the Biological and Social Realm: New Avenues of Inquire. // Symmetry : Culture and Science, (in press).

Marijuán, P. C. (1996b). First Conference on Foundations of Information Science. // Biosystems. 38 (1996) 87-96.

Margalef, R. (1995). La Ecología, entre la vida real y la física teórica. // Investigación y Ciencia. 225 (1995) 66-73.

Nicolis, G. ; Prigogine, I. (1989). Exploring complexity. New York : Freeman, 1986.

Oswald, J. (1986). Théorie de l'information ou Analyse Diacritique des Systèmes. Paris : Masson, 1986.

Popper, R. ; Eccles, J.(1993). El Yo y su Cerebro. Barcelona : Labor, 1993.

Poundstone, W. (1985). The Recursive Universe. Chicago : Contemporary Books, 1985.

Prigogine , I. ; Stengers, I. (1985). Order out of Chaos. London : Flamingo/Fontana, 1985.

Qvortrup, L. (1993). The Controversy over the concept of Information. // Cybernetics and Human Knowing, $1: 4$ (1993) 13.

Rosen, R. (1993). La pregunta de Schrödinger ¿Qué es la vida? 50 años después. // LLUL. 16 (1993) 285-312.

Schrödinger, E. (1944). What's Life: Cambridge, UK : Cambridge University Press, 1967.

Shannon, C.E. ; Weaver, W. (1964).The Mathematical Theory of Communication.. Urbana, Illinois : University of Illinois Press, 1964.

Stonier, T. (1990). Information and the Internal Structure of the Universe. London : Springer Verlag, 1990.

Scire. 2 : 1 (en.-jun. 1996). 
Stonier, T. (1996). La información y la estructura interna del Universo. Barcelona : Ed. Hacer, 1996.

Welch, G. R. (1992). An Analogical "Field" Construct in Cellular Biophysics: History and Present Status. // Prog. biophys. Molec. Biol. 57 (1992) 71-128.

Wicken, J. (1987). Entropy and Information: suggestions for a common lenguage. // Philos. Sci. 54 (1987) 176-193.

Scire. 2 : 1 (en.-jun. 1996). 